\title{
Evaluation of Giardia duodenalis viability after metronidazole treatment by flow cytometry
}

\author{
Joana Barbosa ${ }^{1,2}{ }^{+}$, Acácio Gonçalves Rodrigues ${ }^{1,2}$, Maria José Pérez ${ }^{3}$, Cidália Pina-Vaz ${ }^{1,2,4}$ \\ ${ }^{1}$ Serviço de Microbiologia ${ }^{2}$ Centro de Investigação em Tecnologias e Sistemas de Informação em Saúde, Faculdade de Medicina, \\ Universidade do Porto, Porto, Portugal ${ }^{3}$ Faculdade de Ciências, Universidade de Vigo, Vigo, Galiza, Espanha \\ ${ }^{4}$ Departamento de Microbiologia, Centro Hospitalar de São João, Porto, Portugal
}

Giardia duodenalis (syn. lamblia; syn. intestinalis) susceptibility testing is not routinely performed because the classical culture methods are very time-consuming and laborious. We developed a novel flow cytometry (FC) assay to evaluate the susceptibility of G. duodenalis trophozoites to metronidazole (MTZ). Different concentrations of MTZ were added to cultures of trophozoites $\left(10^{5} / \mathrm{mL}\right)$ and the cultures were incubated for different periods. The $50 \%$ inhibitory concentration was calculated and propidium iodide (PI) was used to quantify the number of dead cells. After treatment, PI-positive trophozoites increased with increasing drug concentration and exposure time. An excellent correlation was found between FC and the classical method. A novel, accurate and reliable method is now available to evaluate $\mathrm{G}$. duodenalis viability.

Key words: Giardia duodenalis - flow cytometry - susceptibility

Giardia duodenalis (syn. lamblia; syn. intestinalis) is the species most frequently responsible for causing diarrhoeal disease in humans (Fricker et al. 2004). Metronidazole (MTZ) is the most commonly used antiparasitic agent for the treatment of infected patients because it prevents trophozoite adhesion to the gastrointestinal mucosa, an important requisite for the establishment of the infectious cycle (Busatti et al. 2007).

The axenic cultivation of Giardia sp., first described in 1970, involves the use of TYI-SS-33 culture medium (Li et al. 2009). However, it is known that several factors could seriously influence cellular growth, e.g., small variations in culture media compounds (trypticase, yeast extract and serum), distilled water quality or culture tube material, which can result in decreased or absent growth and cause microbial contamination ( $\mathrm{Li}$ et al. 2007). Classical susceptibility studies are also not usually performed because they are very cumbersome, tedious and timeconsuming (Li et al. 2007, Zheng et al. 2014).

The use of fluorochrome dyes as cellular viability markers is now well recognised and documented. Propidium iodide (PI), a fluorescent probe that is generally excluded from viable cells, can be used to identify dead cells. Regarding Giardia sp., the correlation between PI-positive cells and their inability to excyst or to infect animals has already been shown (Fricker et al. 2004). The aim of this study was to develop a flow cytometric protocol to evalu-

\section{doi: 10.1590/0074-0276140267}

Financial support: FCT (SFRH/BD/44346/2008) (to JB)

Parts of these results were presented as conference proceed-

ings at the 50th ICAAC, San Francisco, USA.

+Corresponding author: gui75@sapo.pt

Received 23 July 2014

Accepted 27 October 2014 ate MTZ activity on trophozoites. The results obtained were compared with the classic in vitro culture method, which analyses the inhibition of trophozoite growth.

During this study, G. duodenalis trophozoites, type strain WB 36012, from the American Type Culture Collection were maintained in axenic culture in TYI-S-33 growth medium in polystyrene screw-cap tubes $\left(\mathrm{Nunc}^{\circledR}\right)$ or culture tubes until use. Fresh stock solutions of MTZ (4.8 mM, Sigma Chemical Corporation, USA) were prepared by dissolving pure MTZ in phosphate-buffered saline $(8 \mathrm{mM} ; \mathrm{pH}$ 7.1). For MTZ treatment, culture tubes containing $10 \mathrm{~mL}$ G. duodenalis culture $\left(10^{5}\right.$ trophozoites/ $\mathrm{mL}$ ) were individually exposed to different drug concentrations ranging from $0.5-150 \mu \mathrm{g} / \mathrm{mL}(0.003-0.9 \mathrm{mM})$ at $37^{\circ} \mathrm{C}$ for different incubation times $(0,3,6,9$ and $18 \mathrm{~h})$.

Next, growth inhibition was assessed using the adherence properties of $G$. duodenalis cells rather than the capacity of the cells to divide by testing the cellular susceptibility to varying concentrations of MTZ. After drug incubation, culture tubes were placed vertically at $4^{\circ} \mathrm{C}$ for $10 \mathrm{~min}$ and centrifuged at $500 \mathrm{~g}$ for $10 \mathrm{~min}$ and then the supernatant was decanted. Subsequently, the number of cells in the pellet was enumerated in a Neubauer chamber and compared with untreated cells (control). The MTZ concentration that inhibited the adherence of trophozoites by $50 \%\left(\mathrm{IC}_{50}\right)$ was calculated.

For flow cytometric viability evaluation, the percentage of PI-positive (PI+) trophozoites was determined by FC analysis, according to our optimised conditions (Barbosa et al. 2007), with and without MTZ exposure. For each experiment, the percentage of untreated trophozoites (PI-) was subtracted from the percentage of PI+ cells after incubation with the drug. The characteristics of the trophozoite suspensions were evaluated on a FACSCalibur Cytometer (standard model; BD Biosciences, Australia) equipped with three photomultiplayers (PMTs) with standard filters (FL1: band pass of 530/30 nm; FL2: band pass of 585/42 nm; FL3: long pass of $650 \mathrm{~nm}$ ) and a $15 \mathrm{~mW}, 488 \mathrm{~nm}$ argon laser. Cell Quest Pro software 

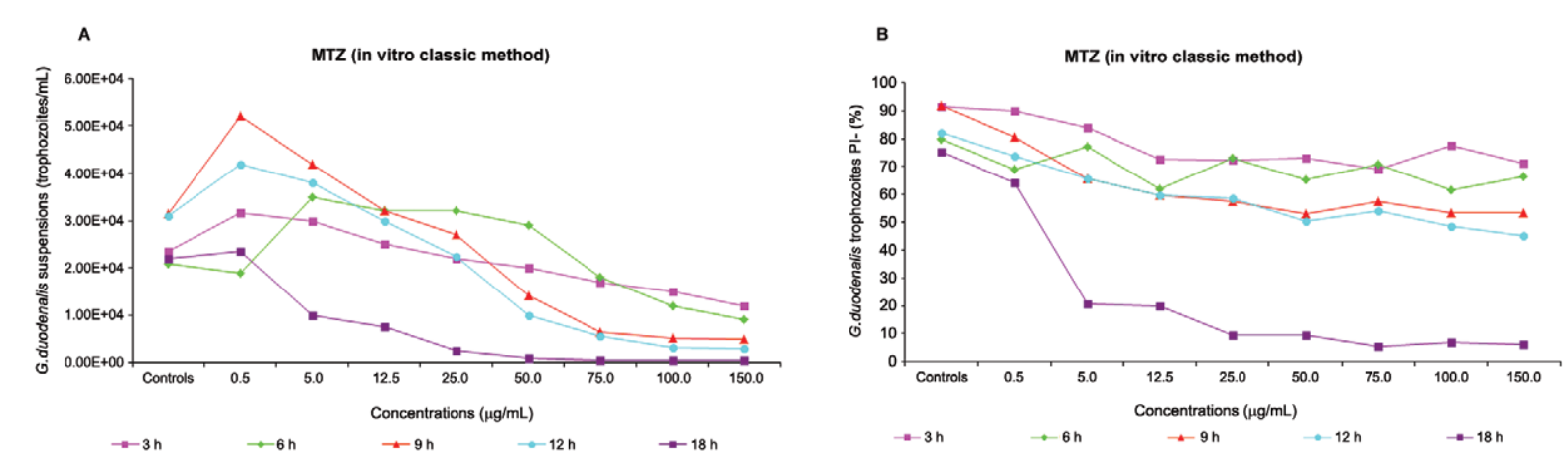

Evaluation of of Giardia duodenalis trophozoite suspensions after treatment with metronidazole (MTZ). A: number of recovered trophozoites evaluated by in vitro classic method; B: percentage of unstained trophozoites with propidium iodide (PI-) evaluated on a FACSCalibur flow cytometer.

v.4.0.2 (BD Biosciences) was used to evaluate the results. Operating conditions included logarithmic $(\log )$ scales on all detectors, namely forward scatter, side scatter and fluorescence detectors. The acquisition settings were defined using an unstained cell sample (autofluorescence) and the PMTs voltage was adjusted to the first $\log$ decade. Instrument controls followed the standard described manufacturer procedures. Data were analysed with SPSS statistical software v.17.0.

Following the experiments, multiple linear regressions were performed to compare the results of FC analysis with the classic in vitro culture method, which was considered to be the dependent variable. Time and PI assays were considered to be predictor values and the ANOVA test was used for model explicability determination. Correlation was determined by a statistically significant $p$ value of $<0.05$ with a $95 \%$ confidence interval. An R square value was obtained for all experiments and each assay was performed in triplicate.

Cellular viability is important because it is the primary determinant of infectious risk; however, it is difficult to evaluate by the standard methods used in clinical microbiology laboratories. The main reason for the lack of knowledge of G. duodenalis susceptibility is because its culture is difficult to perform and impracticable in the day-to-day works of a diagnostic laboratory. Additionally, some difficulties exist, as follows: (i) bacterial contaminations, which can be a problem despite the use of antibiotics as recommended in the literature, (ii) maintenance of axenic conditions, which are essential for optimal trophozoite growth, (iii) interference due to small variations of the water quality and (iv) microscopic evaluation, which requires experience and presents a considerable degree of subjectivity (Bénéré et al. 2007). In contrast, FC is an easier and more reproducible technique that allows analysis of the drug susceptibility of a large number of cells in a short period of time. PI is a nucleic acid-binding fluorescent dye that typically only enters putatively dead cells with severely damaged cytoplasmic membranes (Pina-Vaz et al. 2001). Thus, cells that are impermeable to PI are viable and potentially infectious, whereas cells stained with PI are dead. This information is of crucial importance, especially with regards to drug susceptibility.
Regarding the susceptibility studies of $G$. duodenalis trophozoites to MTZ, a statistically significant correlation $(p<0.05)$ between the in vitro culture classic method and FC was observed at most of the incubation times (Table). Increasing drug concentrations and incubation times resulted in decreased numbers of adherent trophozoites and increased PI+ cells (Figure). At the longest incubation time, only $10 \%$ of the cysts were able to excyst, as previously described (Sousa \& Poiares-da-Silva 1999, Cruz et al. 2003, Sandhu et al. 2004). After 18 h, at MTZ concentrations $>5.0 \mu \mathrm{g} / \mathrm{mL}$, more than $90 \%$ of cells were dead and, therefore, lost the capacity to grow; these cells were classified as PI+ (Figure). Additionally, we determined that the $\mathrm{IC}_{50}$ value was $50.0 \mu \mathrm{g} / \mathrm{mL}$ for a $9 \mathrm{~h}$ incubation and $5.0 \mu \mathrm{g} / \mathrm{mL}$ for an $18 \mathrm{~h}$ incubation. Through the obtained results, we showed an excellent correlation between the number of trophozoites counted in suspension and the percentage of PI- cells (Table).

Although FC allows both morphological and functional evaluation and quantification of individual microorganisms and microcolonies, it remains an underutilised method in microbiology in general and in clinical

\section{TABLE}

Statistical analysis of results after metronidazole treatment using the classic in vitro method as the dependent variable

\begin{tabular}{lccc}
\hline & \multicolumn{2}{c}{$\mathrm{IC}_{50}(\mu \mathrm{g} / \mathrm{mL})$} & \\
\cline { 2 - 3 } Hours & $\begin{array}{c}\text { Classic } \\
\text { methods }\end{array}$ & $\mathrm{FC}$ & $\mathrm{p}$ \\
\hline 3 & 150.0 & 150.0 & 0.03 \\
6 & 100.0 & 150.0 & 0.459 \\
9 & 50.0 & 50.0 & 0.044 \\
12 & 25.0 & 25.0 & 0.004 \\
18 & 5.0 & 5.0 & 0.001 \\
\hline
\end{tabular}

values are considered statistically significant with a $\mathrm{p}<0.05$ and a confidence interval of $95 \%$. FC: flow cytometry; $\mathrm{IC}_{50}$ : inhibitory concentration at $50 \%$. 
microbiology in particular. Our research group has been exploring microbiological applications of FC, namely in the detection and antimicrobial susceptibility evaluation of Mycobacterium tuberculosis and fungi to increase the diagnostic sensitivity and information about clinical samples and isolates with significant advantages (Pina-Vaz et al. 2005a, b). Additionally, several detection protocols for emergent protozoan parasites (Cryptosporidium parvum, $G$. duodenalis) and for fungi (Pneumocystis jirovecii, Encephalitozoon intestinalis) have been developed (Barbosa et al. 2007, 2008, 2009, 2013). Although FC currently requires expensive equipment, this equipment is already present in many immunology laboratories and can often be shared by microbiologists. With this study, we found an alternative method for the evaluation of drug treatment; flow cytometric methods based on fluorogenic assays provide a more accurate, faster, simpler and more automated analysis compared with the classic in vitro method and represent a valid substitute for the assessment of $G$. duodenalis trophozoite viability.

\section{ACKNOWLEDGEMENTS}

To Marisa Machado and Maria Céu Sousa, from Faculdade de Farmácia, Universidade de Coimbra, for all the laboratory assistance and to the kind gift of strain WB 36012 of $G$. duodenalis trophozoites.

\section{REFERENCES}

Barbosa J, Costa-de-Oliveira S, Rodrigues AG, Pina-Vaz C 2007. Optimization of a flow cytometry protocol for detection of Cryptosporidium parvum in hospital tap water and human stools. Int $J$ Antimicrob Agents 29 (Suppl.): S125.

Barbosa J, Costa-de-Oliveira S, Rodrigues AG, Pina-Vaz C 2008. Optimization of a flow cytometry protocol for detection and viability assessment of Giardia lamblia. Travel Med Infect Dis 6: 234-239.

Barbosa J, Costa-de-Oliveira S, Silva AT, Rodrigues AG, Pina-Vaz C 2013. Specific detection of Pneumocystis jirovecii on clinical samples by flow cytometry. Methods Mol Biol 968: 203-211.

Barbosa J, Rodrigues AG, Pina-Vaz C 2009. Cytometric approach for detection of Encephalitozoon intestinalis an emergent agent. Clin Vaccine Immunol 16: 1021-1024.
Bénéré E, da Luz RA, Vermeersch M, Cos P, Maes L 2007. A new quantitative in vitro microculture method for Giardia duodenalis trophozoites. J Microbiol Methods 71: 101-106.

Busatti HGNO, Vieira AED, Viana JC, Silva HE, Souza-Fagundes EM, Martins-Filho OA, Alves RJ, Gomes MA 2007. Effect of metronidazole analogues on Giardia lamblia cultures. Parasitol Res 102: 145-149.

Cruz A, Sousa MI, Azeredo Z, Leite E, de Sousa JCF, Cabral M 2003. Isolation, excystation and axenization of Giardia lamblia isolates: in vitro susceptibility to metronidazole and albendazole. $J$ Antimicrob Chemother 51: 1017-1020.

Fricker CR, Medema GD, Smith HV 2004. Protozoan parasites (Cryptosporidium, Giardia, Cyclospora). In Guidelines for drinkingwater quality, WHO, Geneva, p. 70-118.

Li D, Craik SA, Smith DW, Belosevic M 2007. Comparison of levels of inactivation of two isolates of Giardia lamblia cysts by UV light. Appl Environ Microbiol 73: 2218-2223.

Li D, Craik SA, Smith DW, Belosevic M 2009. Infectivity of Giardia lamblia cysts obtained from wastewater treated with ultraviolet light. Water Res 43: 3037-3046.

Pina-Vaz C, Costa-de-Oliveira S, Rodrigues AG 2005a. Safe susceptibility testing of Mycobacterium tuberculosis by flow cytometry with the fluorescent nucleic acid stain SYTO 16. J Med Microbiol 54: 77-81.

Pina-Vaz C, Costa-de-Oliveira S, Rodrigues AG, Espinel-Ingroff A 2005b. Comparison of two probes for testing susceptibilities of pathogenic yeasts to voriconazole, itraconazole and caspofungin by flow cytometry. J Clin Microbiol 43: 4674-4679.

Pina-Vaz C, Sansonetty F, Rodrigues AG, Costa-de-Oliveira S, Tavares C, Martinez-de-Oliveira J 2001. Cytometric approach for a rapid evaluation of susceptibility of Candida strains to antifungals. Clin Microbiol Infect 7: 609-618.

Sandhu H, Mahajan RC, Ganguly NK 2004. Flow cytometric assessment of the effect of drugs on Giardia lamblia trophozoites in vitro. Mol Cell Biochem 265: 151-160.

Sousa MC, Poiares-da-Silva J 1999. A new method for assessing metronidazole susceptibility of Giardia lamblia trophozoites. Antimicrob Agents Chemother 43: 2939-2942.

Zheng GX, Zhang XM, Yang YS, Zeng SR, Wei JF, Wang YH, Li YJ 2014. An integrated microfludic device for culturing and screening of Giardia lamblia. Exp Parasitol 137: 1-7. 